\title{
The Relationship between Serum Amyloid A Level and Cognitive Dysfunction in Patients with Vascular Dementia: Preliminary Findings
}

\author{
Min Xu $\mathbb{D}^{1},{ }^{1}$ Xiao-ying He $\mathbb{D}^{2},{ }^{2}$ and Pan Huang $\mathbb{D}^{3}$ \\ ${ }^{1}$ Department of Neurology, The Second People's Hospital of Deyang City, No. 340 Minjiang West Road, Deyang, \\ Sichuan 618000, China \\ ${ }^{2}$ Department of Neurology, The Affiliated Hospital of Southwest Medical University, No. 25 Taiping Street, Luzhou, \\ Sichuan 646000, China \\ ${ }^{3}$ Department of Neurology, People's Hospital of Deyang City, No. 173 TaiShan North Road, Deyang, Sichuan 618000, China
}

Correspondence should be addressed to Pan Huang; 1032857970@qq.com

Received 2 January 2021; Revised 25 January 2021; Accepted 3 February 2021; Published 16 February 2021

Academic Editor: Yuzhen Xu

Copyright ( $2021 \mathrm{Min}$ Xu et al. This is an open access article distributed under the Creative Commons Attribution License, which permits unrestricted use, distribution, and reproduction in any medium, provided the original work is properly cited.

\begin{abstract}
Objective. This study was aimed at investigating the relationship between serum amyloid A (SAA) levels and cognitive dysfunction in patients with vascular dementia (VAD). Methods. Using cross-sectional research methods, 146 patients with VAD were selected as the VAD group and 70 normal people were selected as the NC group. Upon admission, the clinical and biochemical characteristics of the two groups of study subjects were collected, and the MMSE scale was used to assess cognitive function. A sandwich enzyme-linked immunosorbent assay was used to detect SAA levels. Results. There was no significant difference in clinical data and biochemical characteristics in the VAD group $(p>0.05)$. Compared with the VAD group, the NC group has a higher level of education $(p<0.05)$. The SAA level of the VAD group was higher than that of the NC group, and there was a significant difference $(p<0.05)$. Spearman correlation analysis showed that SAA and MMSE in the VAD group were negatively correlated. Further multiple regression analysis showed that the serum amyloid A level is an independent risk factor for cognitive dysfunction in VAD patients. Conclusion. The level of SAA in VAD patients is significantly increased, which can be used as a potential peripheral blood marker to predict cognitive impairment in VAD patients.
\end{abstract}

\section{Introduction}

Vascular dementia (VAD) refers to a syndrome with a clinical stroke or subclinical cerebrovascular injury that affects at least one cognitive dysfunction. The disease is a heterogeneous brain disease, accounting for more than $20 \%$ of dementia, and the prevalence of VAD is $1.0 \%-8.8 \%$ [1]. Studies have speculated that by 2030 , VAD patients will reach 66 million, and by 2050, it will reach 120 million $[2,3]$. However, in the face of such a large patient population, there is no specific treatment plan for the disease so far, and there is no specific and sensitive molecular marker [4]. Unlike Alzheimer's disease, VAD is potentially preventable. Finding specific markers that can be detected and diagnosed early is conducive to early intervention, delaying the progression of the disease, and reducing the social burden caused by cerebrovascular disease.

SAA is known for its role in secondary amyloidosis. It was isolated and identified from serum in 1976 and is a highly conserved member of the acute phase protein family [5]. The SAA-encoded protein is located in the p15.1 region of chromosome 11, with a size of $150 \mathrm{~kb}$. Human SAA is synthesized in the liver by the cytokines IL- $1 \beta$, IL- 6 , and tumor necrosis factor- $\alpha$ (TNF- $\alpha$ ) [6]. SAA is the precursor substance of tissue amyloid $\mathrm{A}$ and one of the acute phase response proteins. After the body is infected, wounded, or inflamed, the level of SAA rises rapidly [7, 8]. In recent years, with the in-depth study of SAA's gene regulation, protein structure, and biological function, it has been discovered that SAA is also involved in the pathogenesis of many chronic 
diseases, but the relationship between SAA and VAD is lacking. The main purpose of this study is to detect SAA levels and further clarify whether SAA can be used as a potential biomarker for the prevention and treatment of VAD.

\section{Materials and Methods}

2.1. Research Object. We followed the methods of Dr. Juan Li et al. [9]. A total of $146 \mathrm{VAD}$ patients admitted to our hospital from February 2015 to February 2020 were selected as the VAD group, including 70 females and 76 males, aged 40-75 years, with an average of $69.75 \pm 7.23$ years old. In addition, 70 normal people were selected as the NC group, including 32 females and 38 males, aged 41-78 years old, with an average of $70.31 \pm 6.74$ years old. The diagnosis of VAD refers to the diagnostic criteria established by the National Institute of Neurological Diseases and Stroke (NINDS-AIREN) and the Diagnostic and Statistical Manual of Mental Disorders (DSM-V) and is determined by two neurologists as the diagnosis of VAD $[10,11]$. All VAD patients are diagnosed for the first time and have not been treated. Exclusion criteria are as follows: (1) suffering from other types of dementia; (2) suffering from inflammatory and infectious diseases; (3) suffering from immunological diseases or receiving immunosuppressive treatment; (4) suffering from mental illness; (5) dementia caused by acute cerebrovascular disease or infectious disease; (6) suffering from malignant tumor; (7) operation history or other severe trauma within 3 months; (8) suffering from severe heart, liver, or kidney insufficiency; and (9) used drugs to treat dementia. This study complied with the "Declaration of Helsinki." We obtained the written consent of all subjects and the approval of the Ethics Committee of Deyang People's Hospital.

2.2. Clinical and Biochemical Characteristics. When admitted to the hospital, the investigators collected the clinical and biochemical characteristics of all patients, including years of education, smoking, drinking, high blood pressure (HBP), diabetes (DM), hyperlipidemia (HLP), body mass index (BMI), systolic blood pressure (SBP), diastolic blood pressure (DBP), total cholesterol (TC), triglycerides (TG), high density lipoprotein cholesterol (HDL-C), low density lipoprotein cholesterol (LDL-C), serum creatinine (Scr), and aspartate aminotransferase/alanine aminotransferase (AST/ALT) (Figure 1).

2.3. SAA Determination. In the morning, $5 \mathrm{ml}$ of fasting peripheral venous blood sample was taken from the patient and placed at room temperature to allow it to coagulate naturally for 10-20 minutes. Then, the coagulated blood sample was centrifuged for 30 minutes at $3000 \mathrm{rpm}$. After centrifugation, the upper serum was carefully separated, collected, and stored in a $-80^{\circ} \mathrm{C}$ ultralow temperature refrigerator. SAA was measured from the stored serum using Invitrogen (Waltham, MA, USA) Sandwich ELISA kit according to the manufacturer's instructions.

2.4. Cognitive Function Assessment. The Chinese version of the MMSE scale (Chinese version) was used to evaluate the cognitive function of all patients [12]. The MMSE scale includes six cognitive domains: orientation (time, place),

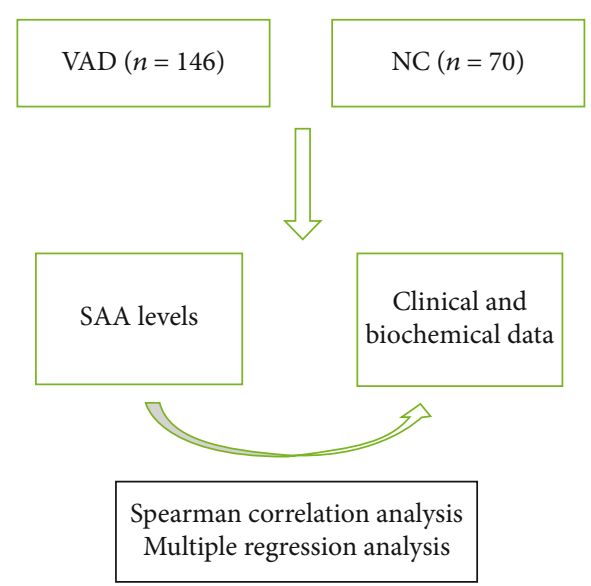

Figure 1: Flow chart.

timely memory, delayed memory, attention and calculation, language ability, and visual space perception. The total score of the scale ranges from 0 to 30 points. $24-30$ is divided into normal, and $<24$ is divided into cognitive dysfunction.

2.5. Statistical Analysis. The concentration of SAA showed a normal distribution. In this study, categorical variables are recorded by numbers (percentage, \%), while continuous variables are recorded by mean \pm standard deviation (mean \pm SD). The $t$-test was applied for the comparison of continuous variables, and the chi-square test was applied for the comparison of categorical variables. Spearman's correlation analysis is used to assess the binary correlation. Multivariate regression analysis was applied to assess the predictive value of clinical and biochemical characteristics on the cognitive function in patients with VAD. The SPSS17.0 statistical software (SPSS Inc., IL, USA) was used in the study, and a $p$ value of 0.05 was considered significant.

\section{Result}

3.1. Clinical and Biochemical Characteristics. The study included a total of 216 patients in the Department of Neurology of our hospital from February 2015 to February 2020, including $146 \mathrm{VAD}$ patients and 70 normal controls. The clinical and biochemical characteristics of all subjects are shown in Table 1. In age, gender, smoke and drinking habits, chronic medical history (HBP, DM, and HLP), and biochemical characteristics (BMI, SBP, DBP,TC, TG, HDL-C, LDL-C, BUA, Scr, and AST/ALT), no significant difference was found between the VAD group and the NC group $(p>0.05)$. However, as shown in Figure 2, there are significant differences between the two groups in terms of years of education, MMSE scores, and SAA levels $(p<0.05)$.

3.2. Spearman Correlation Analysis. Spearman correlation analysis was used to analyze the correlation between clinical and biochemical characteristics and the MMSE score of VAD patients. The results are shown in Table 2 and Figure 3. Spearman correlation analysis showed that the SAA level of VAD patients was negatively correlated with 
TABLE 1: Clinical and biochemical characteristics of all subjects.

\begin{tabular}{|c|c|c|c|}
\hline Item & $\begin{array}{l}\text { VAD group } \\
(n=146)\end{array}$ & $\begin{array}{l}\text { Normal group } \\
\quad(n=70)\end{array}$ & $p$ \\
\hline Age (years) & $69.75 \pm 7.23$ & $70.31 \pm 6.74$ & $>0.05$ \\
\hline $\operatorname{Sex}(M / F)$ & $76 / 70$ & $38 / 32$ & $>0.05$ \\
\hline Smoke $(n, \%)$ & $34(23.28 \%)$ & $17(24.28 \%)$ & $>0.05$ \\
\hline Hypertension $(n, \%)$ & $51(19.02 \%)$ & $15(18.75 \%)$ & $>0.05$ \\
\hline $\mathrm{DM}(n, \%)$ & $25(17.12 \%)$ & $11(15.71 \%)$ & $>0.05$ \\
\hline Drinking habit $(n, \%)$ & $49(33.75 \%)$ & $22(31.43 \%)$ & $>0.05$ \\
\hline $\mathrm{BUA}(\mu \mathrm{mol} / \mathrm{l})$ & $353.85 \pm 34.23$ & $358.21 \pm 35.36$ & $>0.05$ \\
\hline TC (mmol/l) & $4.79 \pm 0.38$ & $4.82 \pm 0.41$ & $>0.05$ \\
\hline $\mathrm{TG}(\mathrm{mmol} / \mathrm{l})$ & $1.25 \pm 0.22$ & $1.28 \pm 0.26$ & $>0.05$ \\
\hline HDL-C (mmol/l) & $0.92 \pm 0.21$ & $0.94 \pm 0.22$ & $>0.05$ \\
\hline LDL-C (mmol/l) & $2.91 \pm 0.42$ & $2.88 \pm 0.43$ & $>0.05$ \\
\hline AST/ALT & $0.73 \pm 0.21$ & $0.74 \pm 0.22$ & $>0.05$ \\
\hline BMI $\left(\mathrm{kg} / \mathrm{m}^{2}\right)$ & $23.54 \pm 1.28$ & $23.74 \pm 1.25$ & $>0.05$ \\
\hline $\mathrm{Scr}(\mu \mathrm{mol} / \mathrm{l})$ & $53.25 \pm 6.47$ & $52.87 \pm 6.35$ & $>0.05$ \\
\hline SBP (mmHg) & $135.62 \pm 15.36$ & $134.82 \pm 15.13$ & $>0.05$ \\
\hline DBP (mmHg) & $88.46 \pm 12.85$ & $87.68 \pm 13.36$ & $>0.05$ \\
\hline $\operatorname{HLP}(n, \%)$ & $30(20.54 \%)$ & $14(20.00 \%)$ & $>0.05$ \\
\hline Education (years) & $10.28 \pm 2.21$ & $14.63 \pm 3.12$ & $<0.05$ \\
\hline MMSE (points) & $28.53 \pm 3.73$ & $21.93 \pm 2.78$ & $<0.05$ \\
\hline SAA $(\mathrm{mg} / \mathrm{l})$ & $99.49 \pm 12.47$ & $17.97 \pm 3.28$ & $<0.05$ \\
\hline
\end{tabular}

NC: normal controls; VAD: vascular dementia; SAA: serum amyloid A; HBP: high blood pressure; SBP: systolic blood pressure; DBP: diastolic blood pressure; HLP: hyperlipidemia; TC: total cholesterol; TG: triglycerides; HDL-C: high-density lipoprotein cholesterol; LDL-C: low-density lipoprotein cholesterol; DM: diabetes mellitus; MMSE: Mini-Mental State Examination; Scr: serum creatinine; BUA: blood urea nitrogen.

the MMSE score, and the correlation coefficient was significant $(r=-0.424, p<0.05)$. However, in our current study, SAA levels are not significantly correlated with the demographic characteristics of VAD patients such as age, gender, education level, smoking habit, drinking habit, HLP, HBP, DM, SBP, DBP, BMI, TC, TG, HDL-C, LDL-C, Scr, BUA, and AST/ALT $(p>0.05)$.

3.3. Multiple Regression Analysis. The results of multiple regression analysis of the MMSE score and SAA level of VAD patients are shown in Table 3. The results show that the SAA level can be used as an independent predictive risk factor for cognitive decline in VAD patients. After adjusting for age, gender, years of education, smoking habit, drinking habit, SBP, DBP, TC, TG, HDL-C, LDL-C, FBG, and other clinical and biochemical characteristics, SAA levels are still important for the independent value of VAD cognitive function significance $(\beta=0.427, p=0.024)$.

\section{Discussion}

The current diagnosis of VAD must meet the following conditions: (1) there must be clinical symptoms of dementia, (2) there must be sufficient evidence of cerebrovascular disease at the same time (including evidence of medical history, physical examination, and radiographic imaging), and (3) the two must be related to each other. In the early 1990s, the diagnostic criteria for $\mathrm{VAD}$ were mainly based on $\mathrm{AD}$, emphasizing irreversible cognitive dysfunction and impaired ability of daily living [13]. However, as research progresses, the definition of VAD is considered to be limited because it does not take into account the more common cognitive impairments associated with cerebrovascular diseases, such as executive dysfunction and psychomotor retardation. Therefore, vascular cognitive impairment (VCI) is introduced; VCI refers to a syndrome with a clinical stroke or subclinical cerebrovascular injury that affects at least one cognitive dysfunction, and VAD is the most serious form of VCI [14]. The main purpose of introducing VCI is to better reflect all cognitive changes caused by vascular factors. It is hoped that the vascular factors of cognitive impairment can be identified early and the vascular risk factors can be controlled to slow down the progression of the disease.

This study investigated the relationship between cognitive function and SAA levels in VAD patients and found that compared with normal controls, VAD patients had higher SAA levels and lower MMSE scores. At the same time, it was also found that the SAA level and MMSE score were significantly negatively correlated in VAD patients, but this correlation was not affected by interfering factors such as age, gender, education level, SBP, DBP, BMI, TG, TC, HDL-C, and LDL-C impact. To our knowledge, this is the first time that SAA is an independent risk factor for cognitive impairment in VAD patients. The pathogenesis of VAD is not clear. Vascular mechanisms, decreased cerebral perfusion, small vessel disease, microinfarction, and microhemorrhage are all related to it. Therefore, the factors that can affect the above links may cause the occurrence of VAD [15-18]. The SAA coding genes include SAA1, SAA2, SAA3, and SAA4, mainly SAA1 and SAA2; both genes are $15-20 \mathrm{~kb}$ in size, and their promoter region contains nuclear factor- $\kappa \mathrm{B}$ (NF- $\kappa \mathrm{B}$ ) and interleukin-6 (IL-6). Transcription factor recognition sequence, which is the binding site of RAS activator, can be activated by interleukin- $1 \beta$ (IL-1 $\beta$ ), IL-6, and tumor necrosis factor (TNF). From the two coding genes, it can be speculated that the biological function of SAA is related to inflammation. Studies have shown that when the concentration of SAA is as low as $10 \mathrm{mg} / \mathrm{l}$, it has the ability to induce chemokines and chemotactic leukocyte migration. When the concentration of SAA is greater than $10-60 \mathrm{mg} / \mathrm{l}$, it can stimulate endothelial cell proliferation, adhesion, invasion, and formation of a capillary-like structure that stimulates the formation of new blood vessels in the body $[19,20]$. In addition, SAA also regulates the outflow or inflow of cholesterol from cells [21]. Current research shows that SAA is related to various pathophysiological processes of atherosclerosis. SAA can stimulate the proliferation and migration of smooth muscle cells, chemoattract neutrophils and monocytes, promote local inflammation, and induce endothelial dysfunction [22]. SAA also increases the synthesis of proteoglycans in vitro and in vivo by inducing transforming growth factor- $\beta$ (TGF- $\beta$ ), leading to increased LDL retention in 

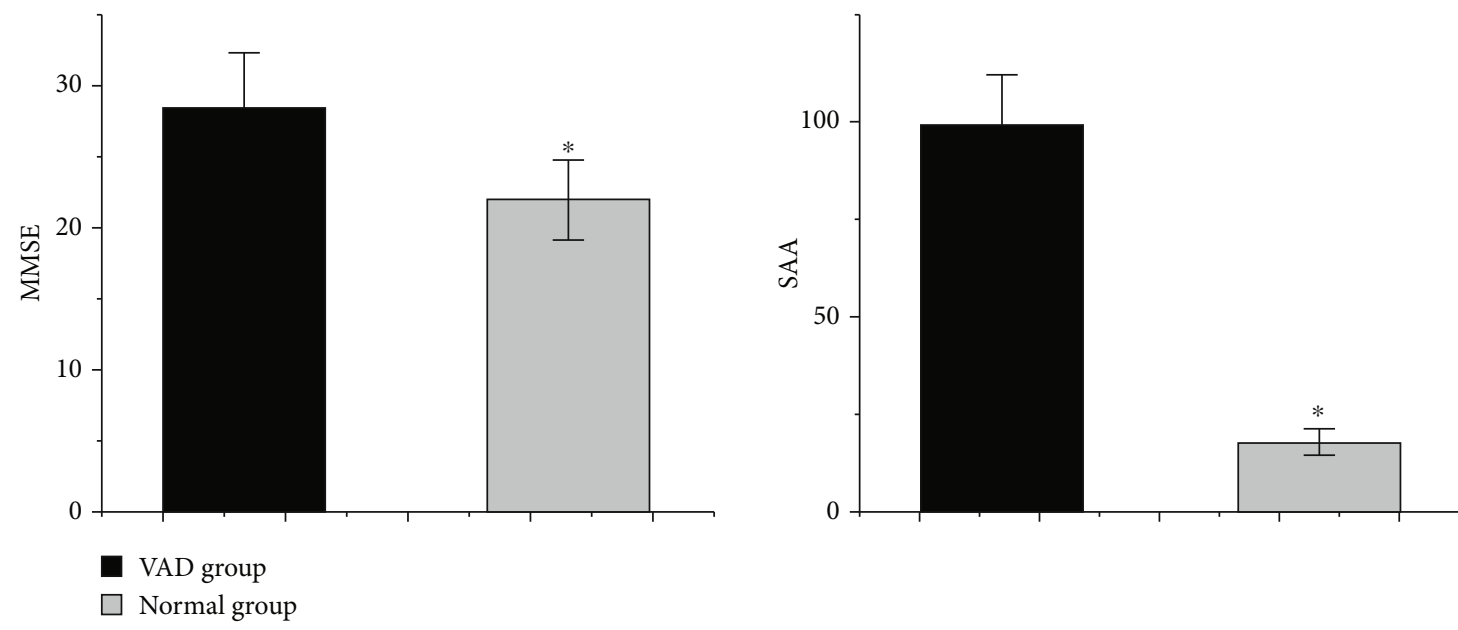

FIgURE 2: Comparison of MMSE and SAA between VAD group and NC group ${ }^{*} p<0.05$.

TABLE 2: Correlation analysis of MMSE score and various parameters in VAD patients.

\begin{tabular}{cccc}
\hline & \multicolumn{3}{c}{ SAA } \\
& $r$ & $p$ \\
\hline MMSE & -0.67 & $<0.05$ \\
\hline
\end{tabular}

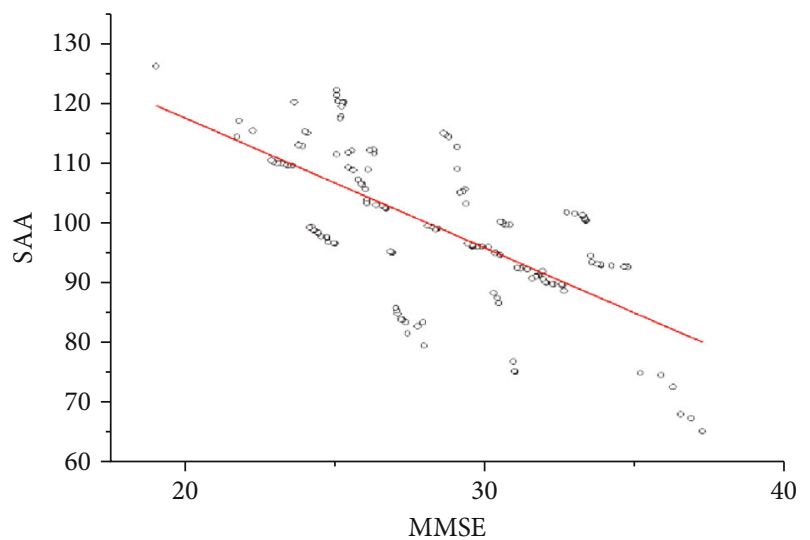

FIgURE 3: Correlation analysis of MMSE score and various parameters in VAD patients.

atherosclerotic lesions [23]. In addition, SAA stimulates the formation of foam cells by inducing the endothelial receptors of oxidized low-density lipoproteins and produces a large amount of lipid peroxides. It can also compete with apolipoprotein A1 to bind to HDL, reducing cholesterol transport to the liver and causing lipid deposition [24]. In addition to being related to atherosclerosis, SAA is also related to obesity and type 2 diabetes. There is a large amount of SAA-mRNA expression in liver fat cells of obese patients, and its expression is related to BMI [25]. In type 2 diabetes, the level of SAA is significantly higher than normal, which is mainly related to the fact that SAA can cause insulin resistance, affect the reverse transport of cholesterol, and stimulate mononuclear
TABLE 3: Relationship between MMSE score and various parameters in VAD.

\begin{tabular}{lccc}
\hline & Regression coefficient & $p$ & $95 \%$ CI \\
\hline Age (years) & 0.139 & 0.176 & $0.724-1.205$ \\
Sex $(\mathrm{M} / \mathrm{F})$ & 0.256 & 0.195 & $0.785-1.189$ \\
SBP $(\mathrm{mmHg})$ & 0.384 & 0.433 & $0.782-1.089$ \\
DBP $(\mathrm{mmHg})$ & 0.376 & 0.335 & $0.551-1.231$ \\
BUA $(\mu \mathrm{mol} / \mathrm{l})$ & 0.213 & 0.186 & $0.736-1.154$ \\
TC $(\mathrm{mmol} / \mathrm{l})$ & 0.271 & 0.178 & $0.826-1.214$ \\
TG $(\mathrm{mmol} / \mathrm{l})$ & 0.312 & 0.569 & $0.482-1.332$ \\
HDL-C $(\mathrm{mmol} / \mathrm{l})$ & 0.406 & 0.226 & $0.779-1.165$ \\
LDL-C $(\mathrm{mmol} / \mathrm{l})$ & 0.263 & 0.185 & $0.825-1.127$ \\
AST/ALT & 0.446 & 0.202 & $0.694-1.098$ \\
BMI $\left(\mathrm{kg} / \mathrm{m}^{2}\right)$ & 0.341 & 0.527 & $0.476-1.128$ \\
Scr $(\mu \mathrm{mol} / \mathrm{l})$ & 0.336 & 0.428 & $0.628-1.257$ \\
Education $($ years $)$ & 0.224 & 0.076 & $0.652-1.183$ \\
SAA $(\mathrm{mg} / \mathrm{l})$ & 0.427 & 0.024 & $1.635-2.854$ \\
\hline
\end{tabular}

macrophages to damage vascular endothelium [26-30]. As we all know, atherosclerosis, obesity, and type 2 diabetes are all risk factors for VAD.

Our study found the differential expression of SSA levels in VAD patients for the first time and confirmed that SAA levels are negatively correlated with the cognitive function of VAD patients, which has important clinical potential application value in VAD. But our research also has some limitations. First, the current study is a single-center study with a small sample. Secondly, we did not monitor SAA levels longitudinally nor did we dynamically follow-up patients' cognitive function and prognosis. Third, because the gold standard for diagnosing VAD is biopsy, our diagnosis of $\mathrm{VAD}$ may not be accurate enough, and VAD patients may also be accompanied by other types of cognitive impairment. Therefore, larger samples of clinical and basic research are needed for further verification. 


\section{Data Availability}

All data, models, and codes generated or used during the study appear in the submitted article.

\section{Ethical Approval}

The study was approved by the Ethics Committee of Deyang People's Hospital, ethics approval number 2014-12-03.

\section{Conflicts of Interest}

The authors declare that they have no any conflict of interests.

\section{Authors' Contributions}

Pan Huang and Xiao Ying He are co-first authors, and they contributed equally to this work.

\section{Acknowledgments}

All the authors are grateful to the Department of Neurology, People's Hospital of Deyang City.

\section{References}

[1] P. B. Gorelick, A. Scuteri, S. E. Black et al., "Vascular contributions to cognitive impairment and dementia," Stroke, vol. 42, no. 9, pp. 2672-2713, 2011.

[2] Q. Wang, Y. Xu, C. Qi, A. Liu, and Y. Zhao, “Association study of serum soluble TREM2 with vascular dementia in Chinese Han population," The International Journal of Neuroscience, vol. 130, no. 7, pp. 708-712, 2020.

[3] Y. Xu, Q. Wang, R. Cui, K. Lu, Y. Liu, and Y. Zhao, "Uric acid is associated with vascular dementia in Chinese population," Brain and Behavior, vol. 7, no. 2, p. e00617, 2017.

[4] J. B. Toledo, S. E. Arnold, K. Raible et al., "Contribution of cerebrovascular disease in autopsy confirmed neurodegenerative disease cases in the National Alzheimer's Coordinating Centre[J]," Brain A Journal of Neurology, vol. 136, no. 9, pp. 2697-2706, 2013.

[5] P. Woo, J. Sipe, C. A. Dinarello, and H. R. Colten, "Structure of a human serum amyloid A gene and modulation of its expression in transfected L cells," The Journal of Biological Chemistry, vol. 262, no. 32, pp. 15790-15795, 1987.

[6] I. N. Baranova, A. C. P. Souza, A. V. Bocharov et al., "Human SR-BII mediates SAA uptake and contributes to SAA proinflammatory signaling in vitro and in vivo," PLoS One, vol. 12, no. 4, article e0175824, 2017.

[7] M. Buck, M. Gouwy, J. Wang et al., "Structure and expression of different serum amyloid A (SAA) variants and their concentration dependent functions during host insults," Current Medicinal Chemistry, vol. 23, no. 17, pp. 1725-1755, 2016.

[8] L. Sun and R. D. Ye, "Serum amyloid A1: structure, function and gene polymorphism," Gene, vol. 583, no. 1, pp. 48-57, 2016.

[9] J. Li, S. Li, Y. Song et al., "Association of serum FAM19A5 with cognitive impairment in vascular dementia," Disease Markers, vol. 2020 , no. 8, 2020.
[10] T. Erkinjuntt, "Clinical criteria for vascular dementia: the NINDS-AIREN criteria," Dementia and Geriatric Cognitive Disorders, vol. 5, no. 1, pp. 189-192, 2004.

[11] M. Heyck and A. Ibarra, "Microbiota and memory: a symbiotic therapy to counter cognitive decline?," Brain Circulation, vol. 5, no. 3, pp. 124-129, 2019.

[12] R. Cassani, M. Estarellas, R. San-Martin, F. J. Fraga, and T. H. Falk, "Systematic review on resting-state EEG for Alzheimer's disease diagnosis and progression assessment," DiseaseMarkers, vol. 5174815, 2018.

[13] G. C. Roman, T. K. Tatemichi, T. Erkinjuntti et al., "Vascular dementia: diagnostic criteria for research studies: report of the NINDS-AIREN International Workshop," Neurology, vol. 43, no. 2, pp. 250-260, 1993.

[14] V. Hachinski, D. Ganten, D. Lackland, R. Kreutz, K. Tsioufis, and W. Hacke, "Implementing the proclamation of stroke and potentially preventable dementias," International Journal of Stroke, vol. 13, no. 8, pp. 780-786, 2018.

[15] A. H. Hainsworth, A. T. Oommen, and L. R. Bridges, "Endothelial cells and human cerebral small vessel disease," Brain Pathology, vol. 25, no. 1, pp. 44-50, 2015.

[16] R. G. de Eulate, I. Goñi, A. Galiano et al., "Reduced cerebral blood flow in mild cognitive impairment assessed using phase-contrast MRI," Journal of Alzheimer's Disease, vol. 58, no. 2, pp. 585-595, 2017.

[17] M. I. Bergkamp, J. G. J. Wissink, E. M. C. van Leijsen et al., "Risk of nursing home admission in cerebral small vessel disease," Stroke, vol. 49, no. 11, pp. 2659-2665, 2018.

[18] J. H. Park, S. W. Seo, C. Kim et al., "Pathogenesis of cerebral microbleeds: in vivo imaging of amyloid and subcortical ischemic small vessel disease in 226 individuals with cognitive impairment," Annals of Neurology, vol. 73, no. 5, pp. 584593, 2013.

[19] M. Connolly, P. R. Rooney, T. McGarry et al., "Acute serum amyloid $\mathrm{A}$ is an endogenous TLR2 ligand that mediates inflammatory and angiogenic mechanisms," Annals of the Rheumatic Diseases, vol. 75, no. 7, pp. 1392-1398, 2016.

[20] C. Hong, C. Shen, H. Ding et al., "An involvement of SR-B1 mediated p38 MAPK signaling pathway in serum amyloid Ainduced angiogenesis in rheumatoid arthritis," Molecular Immunology, vol. 66, no. 2, pp. 340-345, 2015.

[21] R. Kisilevsky and P. N. Manley, "Acute-phase serum amyloid A: perspectives on its physiological and pathological roles," Amy-loid, vol. 19, no. 1, pp. 5-14, 2012.

[22] M. De Buck, N. Berghmans, N. Pörtner et al., "Serum amyloid A $1 \alpha$ induces paracrine IL-8/CXCL8 via TLR2 and directly synergizes with this chemokine via CXCR2 and formyl peptide receptor 2 to recruit neutrophils," Journal of Leukocyte Biology, vol. 98, no. 6, pp. 1049-1060, 2015.

[23] J. C. Thompson, C. Jayne, J. Thompson et al., "A brief elevation of serum amyloid A is sufficient to increase atherosclerosis[S]," Journal of Lipid Research, vol. 56, no. 2, pp. 286293, 2015.

[24] J. M. Wroblewski and A. Jahangiri, "Nascent HDL formation by hepatocytes is reduced by the concerted action of serum amyloid A and endothelial lipase," Journal of Lipid Research, vol. 52, no. 12, pp. 2255-2261, 2011.

[25] R. Z. Yang, M. J. Lee, H. Hu et al., “Acute-phase serum amyloid A: an inflammatory adipokine and potential link between obesity and its metabolic complications," PLoS Medicine, vol. 3, no. 6, article e287, 2006. 
[26] E. Hatanaka, P. T. Monteagudo, M. S. M. Marrocos, and A. Campa, "Interaction between serum amyloid A and leukocytes-a possible role in the progression of vascular complications in diabetes," Immunology Letters, vol. 108, no. 2, pp. 160-166, 2007.

[27] L. Cai, M. C. de Beer, F. C. de Beer, and D. R. van der Westhuyzen, "Serum amyloid A is a ligand for scavenger receptor class B type I and inhibits high density lipoprotein binding and selective lipid uptake*," The Journal of Biological Chemistry, vol. 280, no. 4, pp. 2954-2961, 2005.

[28] J. Y. Mao, J. T. Sun, K. Yang et al., "Serum amyloid A enrichment impairs the anti-inflammatory ability of HDL from diabetic nephropathy patients," Journal of Diabetes and its Complications, vol. 31, no. 10, pp. 1538-1543, 2017.

[29] S. Yuan, K. J. Liu, and Z. Qi, "Occludin regulation of bloodbrain barrier and potential therapeutic target in ischemic stroke," Brain Circulation, vol. 6, no. 3, pp. 152-162, 2020.

[30] Q. Wang, W. Yang, J. Zhang, Y. Zhao, and Y. Xu, “TREM2 overexperession attenuates cognitive deficits in experiment models of vascular dementia," Neural Plasticity, vol. 7, no. $12,2020$. 